УДК 159.9

\title{
Академическая прокрастинация студентов 1 курса: психологические аспекты и особенности
}

\author{
Дарья И. Попова \\ Южный федеральный университет, г. Ростов-на-Дону, Российская Федерация \\ E-mail: darpop@sfedu.ru \\ ORCID ID: https://orcid.org/0000-0001-8675-4775
}

\begin{abstract}
Аннотация
В статье представлены результаты исследования аспектов акалемической прокрастинации у студентов первого курса университета. Во ВвеАении Аано определение акалемической прокрастинации, представлен типичный пример ситуации, в которой находится прокрастинирующий стуАент, привеАены поАХоАы К пониманию природы прокрастинации, обозначены масштабы распространения Аанного явления и его возможные причины. Новизна исследования заключается в изучении аспектов акалемической прокрастинации студентов 1 курса в связи с их смысложизненными ориентациями. В разделе «Методы" описаны основные методики эмпирического исследования: авторская анкета-опросник, «Тест смысложизненных ориентаций» А. А. Аеонтьева, методика «Общий уровень

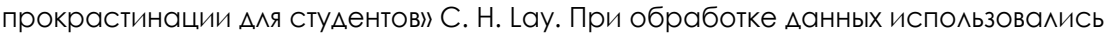
U-критерий Манна-Уитни и корреляционный анализ. В исслеАовании приняли участие 46 обучающихся 1 курса ВУЗа в возрасте 18-21 ^ет. В разАеле «Результатыл представлены результаты изучения особенностей акалемической прокрастинации по пяти направлениям: проявления, причины, эмоциональное сопровожАение, послеАствия А^я учебного процесса и пути преодоления состояния прокрастинации. С помощью U-критерия Манна-Уитни определено отсутствие различий межАу «ситуативными» и "хроническими» прокрастинирующими студентами в связи со смысложизненными ориентациями. Проведение корреляционного анализа показало обратную значимую связь $(-0,338$, при r=0.05) межАу Аанными по опроснику C. H. Lay и общим уровнем осмысленности жизни по методике СЖО. В разделе «ОбсужАение результатов" приведено сравнение полученных результатов с Аанными исследований зарубежных авторов и Аанными нашего предылущего исследования. В Заключении обобщены выводы по результатам исслеАовании и поставлен вопрос об экспериментальном характере будущих исслеАований в Аанном направлении, что Аолжно поспособствовать пониманию природы акалемической прокрастинации у студентов и способов снижения ее влияния на учебный процесс.
\end{abstract}


Innovative Science: psychology. pedagogy. defectology 2021 VOL. 4 \# 4

GENERAL PSYCHOLOGY, PERSONALITY PSYCHOLOGY, HISTORY OF PSYCHOLOGY

\title{
КАючевые слова
}

акалемическая прокрастинация, стулент, эмоции, учебный процесс, университет

\section{Для цитирования}

Попова Д. И. Академическая прокрастинация студентов 1 курса: психологические аспекты и особенности // Инновационная наука: психология, педагогика, дефектология. 2021. Т. 4, No 4. C. 6-26. doi: https://doi.org/10.23947/2658-7165-2021-4-4-6-26

\section{Academic procrastination of 1st-year students: psychological aspects and characteristics}

\author{
Darya I. Popova \\ Southern Federal University, Rostov-on-Don, Russian Federation \\ E-mail: darpop@sfedu.ru \\ ORCID ID: https://orcid.org/0000-0001-8675-4775
}

\begin{abstract}
The article presents the results of a study of aspects of academic procrastination among first-year university students. The Introduction contains the definition of academic procrastination, a typical example of the situation in which a procrastinating student is, approaches to understanding the nature of procrastination, the extent of this phenomenon and its possible causes. The novelty of the research lies in the study of aspects of academic procrastination of 1st year students in connection with their life-meaning orientations. The section "Methods" describes the main methods of empirical research: questionnaire by the author, "Purpose-In-Life Test" by D. A. Leont'ev, "General Procrastination Scale for students" by C. H. Lay. The data were processed using the Mann-Whitney U-test and correlation analysis. The sample included 46 students of the 1st-year of the university at the age of 18-21 years. The section "Results" presents the findings of studying the characteristics of academic procrastination in five directions: manifestations, reasons, emotions, consequences for the educational process, and ways to overcome the state of procrastination. Using the Mann-Whitney U-test, the absence of differences between "situational" and "chronic" procrastinating students was determined in connection with life-meaning orientations. Correlation analysis showed a significant inverse relationship $(-0.338$, at $r=0.05)$ between the data according to the questionnaire by $\mathrm{C}$. $\mathrm{H}$. Lay and the general level of meaningfulness of life according to the PIL test. The section "Discussion of the results" provides a comparison of the results obtained with the data of studies by foreign authors and the data of our previous study. The Conclusion summarizes the findings of our study and raises the question of the experimental nature of future studies in this direction. This should contribute to understanding the nature of students' academic procrastination and how to reduce its impact on the learning process.
\end{abstract}


Инновационная наука: психология. педагогика. дефектология 2021 TОМ 4 № 4

ОБЩАЯ ПСИХОЯОГИЯ, ПСИХОЯОГИЯ ЯИЧНОСТИ, ИСТОРИЯ ПСИХОЯОГИИ

\section{Keywords}

academic procrastination, student, emotions, educational process, university

\section{For citation}

Popova D. I. Academic procrastination of 1st-year students: its psychological aspects and characteristics. Innovative Science: psychology, pedagogy, defectology, 4(4), 6-26. doi: https://doi.org/10.23947/2658-7165-2021-4-4-6-26

\section{Введение}

В нашей работе мы определяем академическую прокрастинацию как:

Академическая прокрастинация - это периодическое систематическое откладывание выполнение академических заданий, сопровождающееся негативными эмоциями и состояниями, способное привести к снижению успеваемости.

Не составит труда описать типичную для «прокрастинирующего» студента ситуацию: после получения задания, выполнение работы откладывается часто до крайних сроков, при этом переживание состояния прокрастинации вызывает сильный стресс, повышает тревожность, из-за чего приступить к выполнению становится еще сложнее. Решающим моментом в начале работы становится наступление момента, когда дадьнейшее откладывание становится невозможным ввиду тяжелых негативных последствий, вероятность наступления которых увеличивается многократно с течением времени. Студенту приходиться делать выбор между сильным напряжением от выполнения заданий и последствиями за их невыполнение (например, потеря стипендии, снижение среднего балиа, необходимого дия участия в конкурсах и т.д.). И хоть студент уже получил тяжелый негативный опыт от прокрастинации, он с большой вероятностью будет и в будущем откладывать выполнение заданий и испытывать это состояние снова и снова.

В литературе академическая прокрастинация рассматривается с нескольких сторон:

1) Как ошибка саморегуляции. Студенты, тодько пришедшие в университет, как правило обладают высокой свободой распоряжения своим временем, т. к. родительский присмотр часто оказывается позади, в школьном периоде обучения. Обязанность регулирования собственного учебного процесса теперь ложится на самого обучающегося. Молодые студенты еще не обучены самостоятельной регуляции, поэтому без внешней поддержки со стороны университета, которая, как правило, сильно ограничена, они начинают испытывать академическую прокрастинацию (Zack \& Hen, 2018). Некоторые люди откладывают действия как неадаптивный образ жизни в раздичных условиях (Steel \& Ferrari, 2013).

2) Как способ справится с негативныли эмоцияли. Академическая прокрастинация предстает как эмоциональная регуляция, которая в краткосрочной перспективе используется для избавления от стресса (Eisenbeck, Carreno \& 
Uclés-Juárez, 2019). Однако здесь наблюдается несоответствие понимания студента своего настоящего «Я» и будущего «Я»: они не сосредотачиваются на будущем, предпочитая переложить имеющиеся задания и переживание ответственности за них на себя будущего (Eisenbeck, Carreno \& Uclés-Juárez, 2019; Sirois, 2014). N. Fiore связывает исподьзование прокрастинации, как механизм преодоления тревоги, с началом или завершением задачи, так и с любыми видами принятия решений (Lenggono \& Tentama, 2020).

3) Как личностную черту. Ученые, работающие в области дифференциальной психологии, воспринимают прокрастинацию как личностную черту, и связывают ее с другими чертами, такими как невротизм и перфекционизм (Hen \& Goroshit, 2020).

Академическая прокрастинация, по данным актуальных исследований, все еще сильно распространена в образоватедьной среде: R. Gurumoorthy и N. S. Kumar указывают на широту распространения прокрастинации от 30 \% до 60 \% среди выпускающихся студентов (Gurumoorthy \& Kumar, 2020). N. Eisenbeck, D. F. Carreno и R. Uclés-Juárez говорят о том, что распространение академической прокрастинации в образовательной среде достигает 90-95 \% (Eisenbeck et al, 2019). В последние несколько десятилетий отмечается значительный рост общей прокрастинации (Eisenbeck et al, 2019).

Причинами академической прокрастинации могут быть: навязанность заданий извне; большие сроки, данные для завершения работы; предполагаемое большое количество времени, которое придется уделить работе; скучные, неприятные или сложные задания; высокая общая занятость (вкдючающая другие задания и дела); новизна задачи; трудностями в принятии решений или начала своей активности; отсроченность получения награды за свои труды; дюбые письменные задания (Попова, 2020). Студентами отмечаются письменные работы как наиболее часто откладываемые виды академических заданий.

В нашем прошлом исследовании мы выделяли вдияние смысловой установки как еще одну возможную причину академической прокрастинации (Попова, 2020). Смысловая установка определяется Д. А. Деонтьевым как «составдяющая исполнительных механизмов деятельности, отражающая в себе жизненный смысл объектов и явлений действительности, на которые эта деятельность направлена, и феноменологически проявляющаяся в раздичных формах воздействия на протекание актуальной деятельности» (Деонтьев, р. 184, 2003). Смысловая установка не осознается субъектом и непроизвольно влияет на его деятельность (Деонтьев, 2003). В соответствии с данными взглядом на прокрастинацию как следствие влияние смысловой установки, прокрастинация будет иметь место, когда активны преградное, отклоняющее и дезорганизующее влияние смысловой установки. При стабилизирующем вдиянии, академическая прокрастинация не должна проявляться (Попова, 2020). 
Цель исследования: изучение аспектов проявления академической прокрастинации студентов 1 курса и эмоциональных состояний, сопровождающих прокрастинацию.

\section{Методы}

Объектом исследования стали студенты Южного федерального университета в количестве 46 человек (из них 39 (84,4\%) женщины и 7 (15,2\%) мужчин), возрастной диапазон составил 18-21 года.

Студенты отвечади на вопросы в период с 10 по 13 июня 2021 года. Сбор ответов осуществлялся с помощью Google Forms. Респонденты принимали участие в опросе добровольно.

Для исследования проявления раздичных аспектов академической прокрастинации при выполнении учебных заданий среди студентов высших учебных заведений была разработана анкета-опросник, вкдючающая 11 утверждений. На основе анализа источников по данной теме были выделены несколько направлений анализа состояния прокрастинации и составлен ряд вопросов, позволяющих изучить специфические особенности академической прокрастинации.

1) Первое направление составила группа вопросов, которые касались особенностей проявления академической прокрастинации. В нее вошли вопросы следующие вопросы: 1) Когда больше всего хочется отдожить выполнение академических заданий?; 2) Когда вы чаще всего приступаете к выполнению задания, которое дается в течение семестра?; 3) Когда вы обычно начинаете подготовку к экзаменам?; 9) Чем вы занимаетесь вместо того, чтобы выподнять важные учебные задания?

2) Второе направление составила группа вопросов, которые касались причин откладывания учебных заданий, это вопросы 4) Из-за чего вы откладываете выподнение задания?; 5) Какие виды заданий вы откдадываете чаще всего?

3) В третьем направлении освещались особенности эмоционального сопровождения состояния прокрастинации, это направление было представлено следующими вопросами: 6) Во время откладывания выполнения заданий, испытываете ли вы какие-дибо негативные эмоциональные состояния?; 7) Если на предыдущий вопрос вы ответили «Да», укажите одно или несколько эмоциональных состояний, которые вы испытываете во время откладывания задания; 8) Бывало ли у вас такое состояние, когда вы уже сели за выполнение задания, но не могли его начать, так как испытывали состояние сильной скованности, ступора?

4) Четвертое направление акцентирует внимание на последствиях академической прокрастинации для учебного процесса. В данном опроснике оно представлено 1 вопросом: 10) Пропускаете ли вы занятия из-за того, что не выполнили задание? 
5) Пятое направление касается представлений о путях преодоления состояния академической прокрастинации и представлено 1 вопросом: 11) Что стимулирует Вас на выполнение заданий?

Респондентам предлагалось выбрать одно из нескольких утверждений, наиболее близкое им по сути, также присутствовали вопросы с множественным вариантом ответа.

Для исследования смысложизненных ориентаций студентов был использован Тест смысложизненных ориентаций Д. А. Деонтьева (Деонтьев, 2000).

Для определения уровня академической прокрастинации была исподьзована методика Общий уровень прокрастинации для студентов C. H. Lay (Lay, 1986).

Дия обработки полученных данных были исподьзованы следующие методы математической статистики: U-критерий Манна-Уитни, Корреляционный анализ.

\section{Результаты}

\section{Анализ состояния прокрастинации студентов 1 курса}

В соответствии с первым направлением исследования - выявлением особенностей академической прокрастинации, были проанализированы вопросы анкеты под номерами 1, 2, 3, 9 .

По вопросу 1 "Когда бодьше всего хочется отдожить выподнение академических заданий?” распределение ответов оказалось следующим: 32,6 \% студентов хочется откладывать выполнений всё время, у 26,1 \% это желание откладывать возникает перед сессией, у 26,1 \% после сессией, у 15,2 \% после каникул. Эти данные могут свидетельствовать о числе “здостных" академических прокрастинаторов, то есть, числе (32,6 \%) тех студентов, которые откладывают выполнение заданий всё время, независимо от внешних факторов.

Показательными являются данные, полученные в ответ на вопрос 2 “Когда вы чаще всего приступаете к выподнению задания, которое дается в течение семестра?", они представлены на рисунке 1. Согласно этим данным 19,6\% студентов откладывают текущие задания до последнего момента, и тодько 15,2 \% приступают к выполнению задания сразу после его получения. Таким образом пятая часть опрошенных студентов склонны выполнять задание непосредственно перед сроком сдачи, что, вероятно, сказывается на качестве задания. 


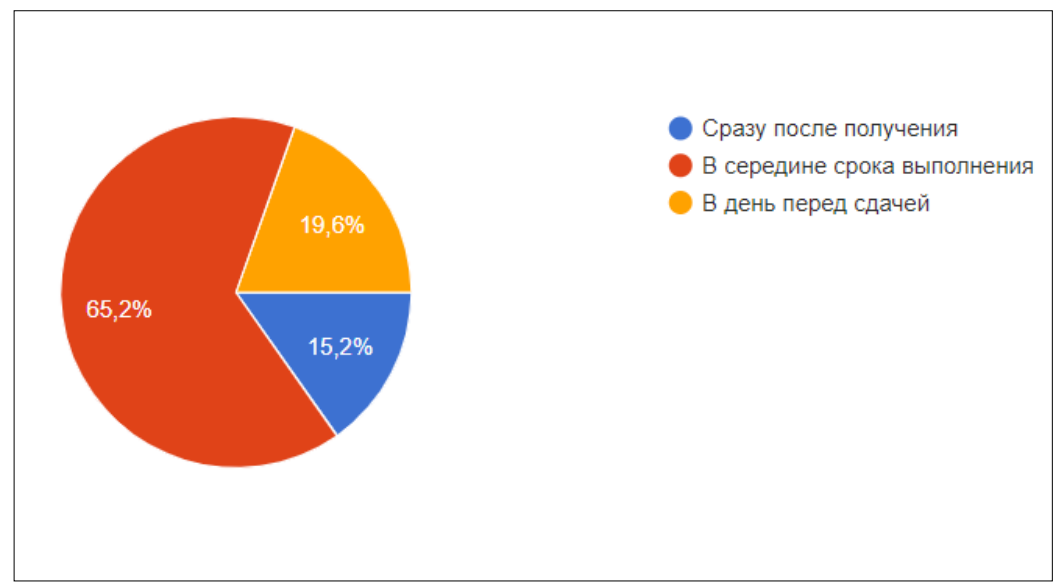

Рисунок 1. Распределение ответов респондентов на вопрос анкеты-опросника № 2 "КогАа вы чаще всего приступаете к выполнению задания, которое дается в течение семестра?"

В вопросе 3 “Когда вы обычно начинаете подготовку к экзаменам?” ответы студентов распределились следующим образом. 58,7\% готовятся к экзаменам в последние дни перед самим экзаменом, 34,8 \% в течение всего семестра, 6,5\% в ночь перед экзаменом. Ни один респондент не отметил, что начинает подготовку с начала семестра. Таким образом, хоть и экзамен и является доводьно значимым академическим событием, однако, тодько примерно треть студентов готовятся к нему заблаговременно.

Важным аспектом изучения академической прокрастинации является анадиз распределения своего времени студентами. Ответы на вопрос 9 “Чем вы занимаетесь вместо того, чтобы выполнять важные учебные задания?" (допускался выбор нескольких вариантов) распределидись следующим образом. Самым популярной альтернативной выполнению академических заданий является занятие любимым делом “(смотрю сериалы, фильмы, видео, играю в видеоигры, читаю книги, слушаю музыку и т. д.)”, ее отметили $63 \%$ респондентов, на втором месте вариант “Занимаюсь домашними делами” - 52,2 \%, на третьем - “Готовдю задания для других предметов, потому что избегаю выполнения другого «мучающего» задания", его отметиди 47,8 \% респондентов, далее по популярности идет вариант “Занимаюсь незначительными (простыми) делами” - 34,8 \%. Для выявления доди студентов, не склонных к прокрастинации был введен вариант “Мне не присуще откладывание выполнения заданий”, его выбрали 6,5 \% респондентов. 
В рамках 2-го направления - изучения причин академической прокрастинации респондентам был задан прямой вопрос: “Из-за чего вы откладываете выполнение задания?”. При ответе на данный вопрос респонденты могди выбрать несколько вариантов ответа. Самыми популярными ответами стали: 1) “Задание воспринимается мной как трудное (трудоемкое, требующее значительных усилий)" - 50 \%, и “Задание воспринимается мной как сложное (непонятно, как его выподнять)” - 50 \%, 3) “Ощущение бессмысленности выполнения этого задания для себя" - 43,5\%. Более подробные данные представлены в Таблице 1.

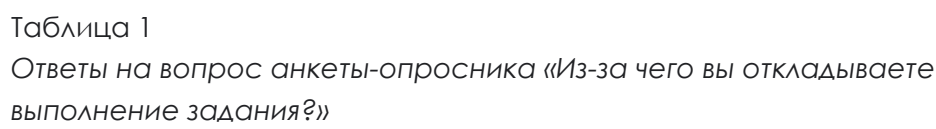

Ощущение бессмысленности выполнения этого заАания Аля себя

Задание воспринимается мной как трудное (трудоемкое, требующее $50 \%$ значительных усилий)

Залание воспринимается мной как сложное (непонятно, как его $50 \%$

выполнять)

Преполаватель, Аавший залание, вызывает негативные эмоции

Большой срок выполнения заАания $34,8 \%$

Неуверенность в собственных способностях

Опасение негативной обратной связи 
Инновационная наука: психология. педагогика. дефектология 2021 TОМ 4 № 4

ОБЩАЯ ПСИХОЯОГИЯ, ПСИХОЯОГИЯ ЯИЧНОСТИ, ИСТОРИЯ ПСИХОЯОГИИ

Для проверки предположения о том, что тип задания может выступать причиной промедления его выполнения был задан вопрос: “Какие виды заданий вы откладываете чаще всего?". Распределение ответов респондентов представлено в Таблице 2.

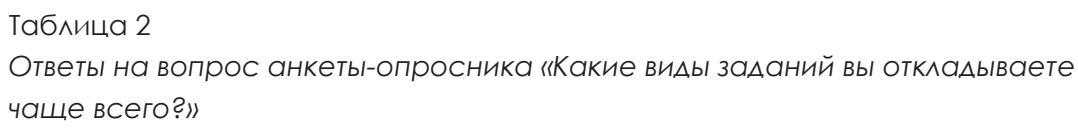

Ответы на вопрос анкеты-опросника «Какие виды заланий вы откладываете чаще всего?»

Вариант ответа

Процент респондентов, выбравших Аанный вариант

Подготовка к семинару $17,4 \%$

Подготовка к лекции $10,9 \%$

Написание письменных работ (эссе, рефрерат) $73,9 \%$

Подготовка к саяче проектных работ $43,5 \%$

Из этих данных следует, что гораздо чаще откладываются задания письменные, предполагающие некоторый продукт, чем те, где необходимо что-дибо прочитать, или отвечать устно.

В рамках 3-го направления исследования были заданы вопросы об эмоциональных состояниях, сопровождающих прокрастинацию. При ответе на вопрос “Во время откладывания выполнения заданий, испытываете ли вы какие-либо негативные эмоциональные состояния?" 78,3 \% выбрали вариант “Да", 21,7\% вариант “Нет. Следующий вопрос “Если на предыдущий вопрос вы ответиди «Да», укажите одно или несколько эмоционадьных состояний, которые вы испытываете во время откладывания задания" предполагал уточнение эмоциональных состояний при академической прокрастинации. Ответы на данный вопрос представлены на Рисунке 2. 
Innovative Science: psychology. pedagogy. defectology 2021 VOL. 4 \# 4

GENERAL PSYCHOLOGY, PERSONALITY PSYCHOLOGY, HISTORY OF PSYCHOLOGY

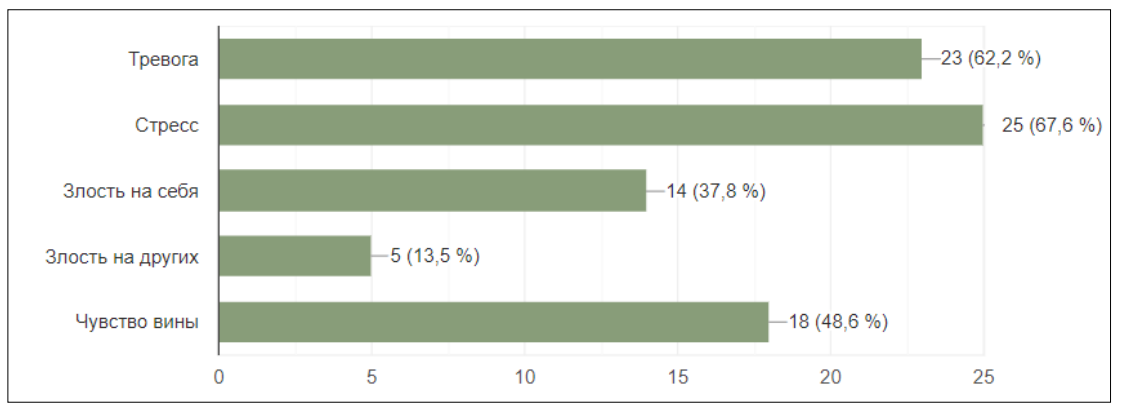

Рисунок 2. Распределение ответов респондентов на вопрос анкеты-опросника № 7 "Укажите одно или несколько эмоциональных состояний, которые вы испытываете во время откладывания задания"

Вопрос анкеты № 8 был направлен на выявление распространенности специфического психофизиологического состояния затруднения при начале выполнения задания, которое мы обозначаем как «прокрастинический ступор». Данные представлены на Рисунке 3.

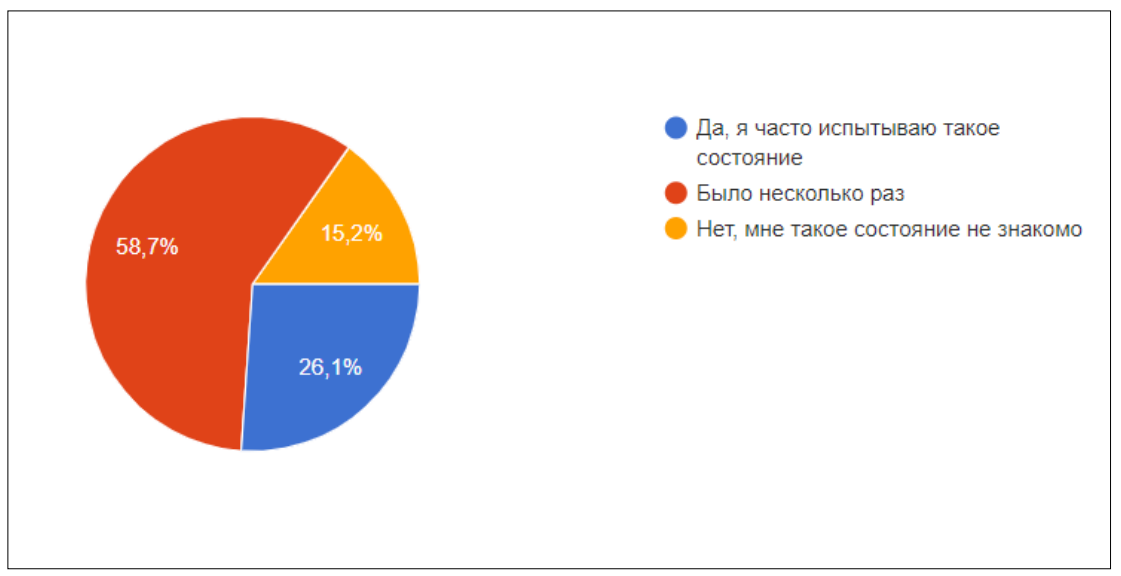

Рисунок 3. Распрелеление ответов респондентов на вопрос анкеты-опросника № 8 "Бывало ^и у вас такое состояние, когАа вы уже сели за выполнение задания, но не могли его начать, так как испытывали состояние сильной скованности, ступора?"

Вопрос анкеты № 10 “Пропускаете ли вы занятия из-за того, что не выполнили задание?" был направлен на выявление актуальности изучения состояния 
Инновационная наука: психология. педагогика. дефектология 2021 TОМ 4 № 4

ОБЩАЯ ПСИХОЯОГИЯ, ПСИХОЯОГИЯ ЯИЧНОСТИ, ИСТОРИЯ ПСИХОЯОГИИ

академической прокрастинации с точки зрения ее вдияния на учебный процесс. 60,9\% респондентов отметили, что не пропускают занятия по этой причине, 37 \% признались, что несколько раз пропускали занятия по этой причине, a 2,2 \% - что часто пропускают занятия по этой причине.

Вопрос анкеты № 11 был направлен на выявление путей преодоления состояния академической прокрастинации. Результаты представлены в таблице 3.

Таблица 3
Ответы на вопрос анкеты «Что стимулирует Вас на выполнение заланий?»

Вариант ответа

Возможные негативные последствия от того, что работа не слана к сроку

Упреки в невыполнении залания

От роАственников или Арузей

ЧувСтво вины Или СтыАа Из-за

того, что выполнение работы

ОткАаАывается

Аавление со стороны

преподавателя

ОАобрение со стороны

родственников или Арузей в случае

выполнения заАания в срок

Желание поскорее «освободиться»

от задания
Процент респондентов, выбравших Аанный вариант
$69,6 \%$

$4,3 \%$

$47,8 \%$

$8,7 \%$

$4,3 \%$

$73,9 \%$

Анализ состояния прокрастинации студентов 1 курса в сравнении с 2-4 курсом

Данные, полученные после прохождения студентами первого курса анкеты-опросника, были сравнены с данными нашего прошлого исследования, где авторскую анкету заполняли студенты 2-4 курсов (Попова, Беликова, 2021). Мы сопоставляли результаты двух исследований чтобы выяснить, есть ди раздичия между студентами первого и более старших курсов. Показательно то, что по большей части вопросов ответы респондентов двух исследований схожи. 
Отличия наблюдаются в вопросах 3, 4, 5, 7 .

В вопросе № 3 «Когда вы обычно начинаете подготовку к экзаменам?» бодьшая часть студентов первого курса, по сравнению с 2-4, начинает подготовку в течении семестра, в то время как студенты старших курсов бодьше полагаются на подготовку в последние дни перед экзаменом. Эти данные представлены на рисунке 4.

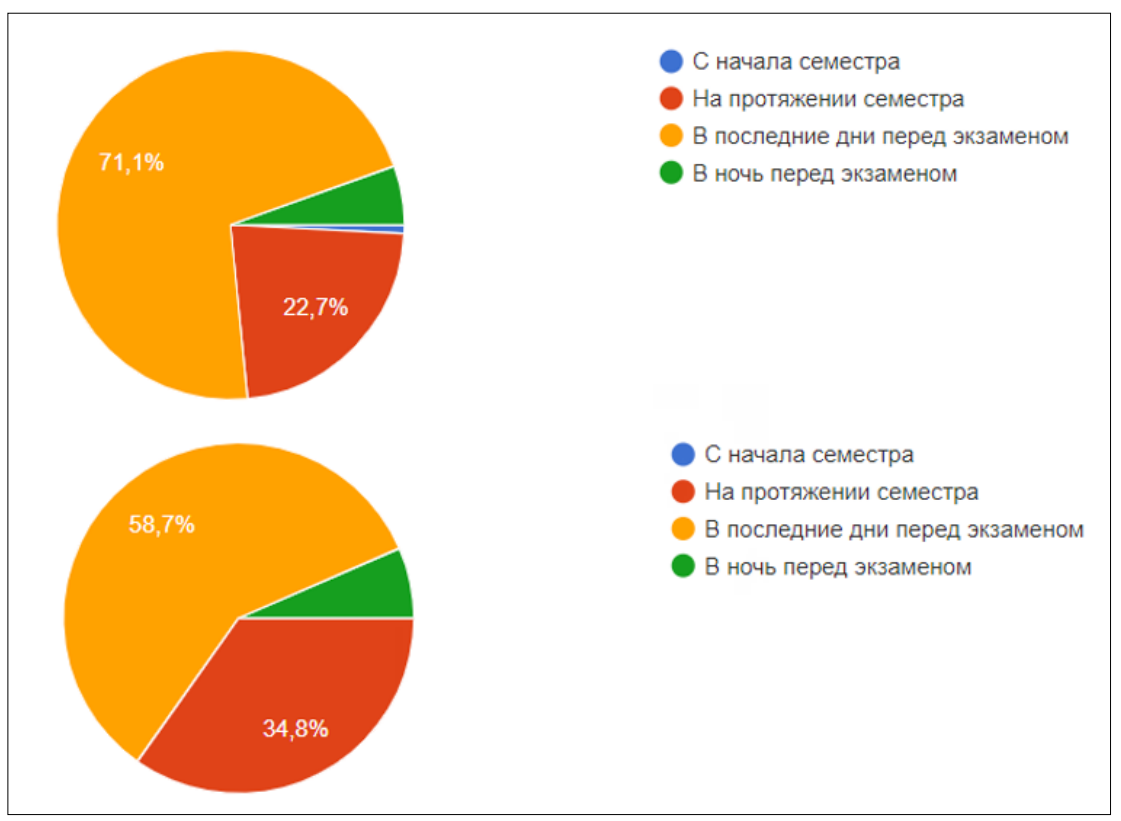

Рисунок 4. Распределение ответов респондентов на вопрос анкеты-опросника

3. КогАа вы обычно начинаете подготовку к экзаменам? Вверху - данные опросника среди студентов 2-4 курсов, внизу-студентов 1 курса.

В вопросе № 5 большая часть студентов первого курса откладывает задание из-за того, что не может понять, как выподнять задание, считает его сложным, а также из-за ощущения бессмысленности его выполнения.

Данные вопроса № 6 дают понять, что студенты 1 курса значительно реже откладывают выполнение проектных работ, чем студенты старших курсов. Это может быть связано с тем, что на старших курсах чаще в качестве академических заданий выступают именно проектные задания.

Студенты первого курса чаще испытывают стресс при откладывании выполнения академических заданий. Студенты 2-4 курса лучше адаптированы 
Инновационная наука: психология. педагогика. дефектология 2021 TОМ 4 № 4

ОБЩАЯ ПСИХОЯОГИЯ, ПСИХОЯОГИЯ ЯИЧНОСТИ, ИСТОРИЯ ПСИХОЯОГИИ

к учебной среде в связи с их опытом, поэтому испытываемого стресса у них меньше. Что примечательно, тревога имеет высокие значения в обоих случаях.

Анализ состояния прокрастинации студентов 1 курса в связи со смысложизненными ориентациями

По вопросам № 1 (когда больше всего хочется откладывать выполнение заданий) и № 10 (пропуск занятий по причине неготового задания) выборка студентов всех курсов была разделена на две половины: условно на студентов, которые иногда находятся в состоянии прокрастинации («ситуативные»), и те, кто находится в этом состоянии перманентно («хронические»). С помощью U-критерий Манна-Уитни мы проанадизировали данные выборки в связи со смысложизненными ориентациями. Значимых раздичий между «ситуативными» и «хроническими» прокрастинаторами не было обнаружено (рис. 6). Мы полагаем, что, несмотря на то, насколько тщательно планирует свое будущее человек, то, насколько осмысленна его жизнь, не связано с тем, насколько сильно он прокрастинирует.

Общий уровень прокрастинации для студентов C. H. Lay и Тест смысложизненных ориентаций Д. А. Леонтьева

Шкала прокрастинации C. H. Lay позволила разделить студентов 1 курса на три группы в зависимости от уровня прокрастинации. По каждому из уровней прокрастинации были посчитаны средние балды, набранные студентами, вошедшими в эти уровни: высокий уровень - 60 баллов, средний уровень -44 балла, низкий уровень - 40 баллов. Распределение уровней прокрастинации по выборке из первокурсников выглядит следующим образом: 38,5\% с высоким уровнем, 41 \% со средним и 20,5\% с низким уровнем прокрастинации.

Корреляционный анализ по данным опросника Lау и методики СЖО показал обратную значимую связь $(-0,338$, при r=0.05). То есть, есть тенденция к тому, что, чем выше уровень осмысленности жизни, тем ниже уровень прокрастинации.

Однако внутри групп с разным уровнем прокрастинации не было найдено значимых раздичий по шкалам методики «СЖО». То есть то, насколько планирует свое будущее человек, то насколько осмысленна его жизнь не связано с тем, насколько сильно он прокрастинирует. Эти данные подтвердиди отсутствие связи уровня осмысленности жизни с вопросами 1 и 11 которые мы упоминали ранее.

\section{Обсуждение резудьтатов}

Результаты нашего исследования показали несколько граней такого явления как академическая прокрастинация среди студентов первого курса. Для них проблема прокрастинации может стоять особенно остро, т. к. как они перешли в новую для себя образовательную среду. S. Y. Lee и N. C. Hall утверждают, что студенты первого курса особенно восприимчивы к академической прокрастинации из-за большого количества открывающихся перед ними новых возможностей 
(например, социализация, волонтерство) и проблем (например, возможные неудачи в учебе и принятие решений, большой автономии), с которыми они сталкиваются при переходе на среднее образование (Lee \& Hall, 2020).

\section{Анализ проявлений состояния прокрастинации}

Наши результаты показывают, что 58,7 \% опрошенных студентов начинают подготовку к экзамену в последние дни перед ним, а 6,5 \% готовятся в ночь непосредственно перед экзаменом. Несмотря на значимость этого академического мероприятия, вдияющего на стипендию, бодьшинство студентов приступают к подготовке к нему доводьно поздно.

Студент может откдадывать подготовку к экзамену из-за самого страха оценивания его знаний и способностей. J. Gil Flores, M. R. De Besa Gutiérrez и A. Garzón Umerenkova отмечают, что касается оценочной тревожности, то есть страха студента перед оценкой его уровня компетентности или успеваемости, а также перед негативными последствиями, которые могут возникнуть из-за этого, то эта тема широко обсуждается в литературе, особенно когда она указывается в тревоге перед тестами и экзаменами (Gil Flores, De Besa Gutiérrez, \& Garzón Umerenkova, 2019).

В случае сдачи заключительных заданий, которым будет выставлена оценка, которая может иметь решающее значение для успешной сдачи предмета, неудивитедьно, что беспокойство перед оценкой приводит к поведению, закдючающемуся в уклонении от задания и задержке. Это одна из главных причин откладывать дела на потом.

\section{Анализ причин появления состояния прокрастинации}

В нашем исследовании такие характеристики задания как «трудное (трудоемкое, требующее значительных усилий)» и «сложное (непонятно, как выполнять это задание) отмечались 50 \% студентов в отношении обоих характеристик. 43,5\% опрошенных студентов выделяди «ощущение бессмысленности выполнения задания» как причину прокрастинации.

Наибольшую прокрастинацию у студентов вызывают задания, при выполнении которых предполагается затрата большого количества усилий и психических ресурсов. Непонимание механизма выполнения задания увеличивает его шанс быть отложенным. J. Gil Flores, M. R. De Besa Gutiérrez и A. Garzón Umerenkova выделяют неадекватные реакции на требования задания как причину прокрастинации (Gil Flores et al, 2019). Ситуация, когда преподаватель не дает четкой инструкции о том, из каких компонентов состоит задание, и как его выполнять, отмечается как потенциально способная вызвать академическую прокрастинацию. Исследования прокрастинации также показывают, что люди с большей вероятностью откладывают неприятные задачи (Milgram, Sroloff \& Rosenbaum, 1988; Blunt \& Pychyl, 2000), или когда вознаграждение за выполнение задач более отдаленное (Steel, 2007; Rahimi, Hall \& Pychyl, 2016). По данным нашего 
исследования 34,8 \% студентов выбирают в качестве причины возникновения прокрастинации большой срок, данный на выполнение заданий. Вопрос связи прокрастинации и сроков выполнения был освящен в работе авторов. Они обнаружили, что прокрастинация усиливается, когда преподаватель снижает уровень спроса, готов оговаривать сроки выполнения заданий и проявляет большую гибкость при выставлении оценок.

Задача откдадывается, когда она воспринимается студентом как важная, стрессовая, сложная и сбивающая с толку (Pychyl, Lee, Thibodeau \& Blunt, 2000), как требующая усилий, провоцирующая беспокойство, и когда она не позволяет студенту продемонстрировать свои навыки и создать уверенность в себе (Ferrari \& Scher, 2000). С другой стороны, если задача воспринимается, как неинтересная, требующая небольшого разнообразия навыков, не вознаграждаемая, не связанная с темой других работ студента и не связанная с нормами, предполагающими быстрое выполнение задач, ее выполнение также будет откладываться (Rahimi et al, 2016). В нашем предыдущем исследовании студенты 2-4 курсов меньше отмечади ощущение бессмысленности выполнения академических заданий как нечто, что мешает выподнению заданий (Попова, Беликова, 2021). Мы предполагаем, что это связано с тем, что на более старших курсах учебная программа содержит больше профильных предметов подготовки, чем на первом курсе.

Аналогичным образом, когда учащиеся воспринимают задачи как сложные и требующие больших усилий, они склонны проявлять более высокий процент откладывания на потом (Ferrari, Mason \& Hammer, 2006). Однако чрезмерно простые задачи могут быть скучными и неинтересными, способствуя прокрастинации, поэтому поставленные задачи должны обеспечивать баланс между их уровнем спроса и возможностью их выполнения учеником (Steel, 2007).

Анализ эмоциий и эмоцчиональных состояний, сопровождающцих состояние прокрастинациии

Состояние прокрастинации сопровождается раздичными негативными эмоциями. В нашем исследовании наиболее часто отмечались тревога, переживание стресса и чувство вины. В своей работе J. Gil Flores, M. R. De Besa Gutiérrez и A. Garzón Umerenkova отмечают, что частая прокрастинация связана с проблемами тревоги, стресса, физического и психического здоровья (Gil Flores et al, 2019). Кроме того, по их данным, прокрастинацию сопровождает чувство разочарования (Gil Flores et al, 2019).

Было обнаружено, когда прокрастинаторы сталкиваются с трудными задачами или ситуациями, они пытаются уменьшить переживание отрицательных эмоций, уходя от ситуации, вызывающей отрицательные эмоции. Откладывая выполнение заданий, они уменьшают влияние негативных эмоций в краткосрочной перспективе, но это не дает им достичь своих целей в долгосрочной перспективе (Bytamar, Saed \& Khakpoor, 2020). 
Innovative Science: psychology. pedagogy. defectology 2021 VOL. 4 \# 4

GENERAL PSYCHOLOGY, PERSONALITY PSYCHOLOGY, HISTORY OF PSYCHOLOGY

В ходе длительного исследования D. M. Тісе и R. F. Baumeister обнаружили, что состояние прокрастинирующих студентов ухудшается к концу семестра по сравнению с началом, а именно обнаруживаются симптомы болезни и стресса (Tice \& Baumeister, 1997).

Анализ влияния прокрастинации на течение учебного процесса

В нашем исследовании 37 \% студентов отметили, что пропускали занятия несколько раз по причине не выподненного задания. Неготовое задание является причиной частых непосещений занятий для 2,2 \% опрошенных студентов.

К концу семестра у откладывающих студентов может скапливаться большое количество задолженностей, которые нужно сдать до начала сессии. Страх негативных последствий из-за несданной работы и чувство вины или стыда наконец пересиливают состояние прокрастинации, и работа над выполнением откладываемых заданий начинается. Однако интенсивная работа в крайне сжатых сроках может негативно сказываться на физическом и психическом здоровье обучающихся. Даже если прокрастинирующему студенту удается уложиться в сроки и закрыть задолженности, он остается эмоционально истощенным к началу сессии или экзаменов, что, во-первых, может сказаться на оценке усвоения пройденного курса, во-вторых, ухудшить эмоциональное состояние обучающегося.

Высокий уровень прокрастинации G. Galić, K. Matešić и L. Pačić-Turk связывают с более низкой успеваемостью. Студенты с более низким уровнем прокрастинации достигают лучших результатов, в то время как хроническое откладывание заданий снижает успеваемость учащихся, что приводит к худшим резудьтатам (Galić, Matešić \& Pačić-Turk, 2019). Студент, приступивший к подготовке к экзамену в последние дни, или в последнюю ночь может прибегать на экзамене к списыванию (Galić et al, 2019).

Анализ путей преодоления студентами состояния прокрастинации

В настоящем исследовании, самые популярные варианты ответов на вопрос № 12 «Что стимулирует Вас на выполнение заданий?» можно объединить общим стремлением избавиться от напряжения, вызванного либо тревогой о последствиях невыподнения задания, дибо переживаниями вины иди стыда перед преподавателем и/иди одногруппниками, дибо же тем фактом, что задание остается невыполненным и тем самым заставляет уделять ему психологические и эмоциональные ресурсы.

Другими словами, вместо того чтобы исподьзовать стратегии адаптивного регулирования эмоций, студенты используют прокрастинацию как способ регулирования своих эмоций; стратегия, которая уменьшает негативные эмоции в краткосрочной перспективе, но не дает им достичь своих целей в долгосрочной перспективе. 
Инновационная наука: психология. педагогика. дефектология 2021 TОМ 4 № 4

ОБЩАЯ ПСИХОЯОГИЯ, ПСИХОЯОГИЯ ЯИЧНОСТИ, ИСТОРИЯ ПСИХОЯОГИИ

Анализ результатов опросников «Общий уровень прокрастинации для студентов» и «Тест смысложизненных ориентаций»

Одним из компонентов осмысленности жизни, измеренным нами по методике смысложизненных ориентаций, является локус контроля. Уровень локуса контроля отражается на легкости/сложности принятия решений, планировании своей будущей деятельности. По нашим данным, уровень осмысленности жизни имеет обратную значимую связь $(-0,338$, при $\mathrm{r}=0.05)$ с уровнем академической прокрастинации. Результаты Р. Е. Morris и С. O. Fritz показали, что откладывание принятия решений и академическая прокрастинация умеренно и положительно связаны (Morris \& Fritz, 2015).

\section{Закдючение}

Применение метода анкетирования в данном исследовании позводило изучить ряд аспектов проявления прокрастинации в том виде, как они воспринимаются студентам. С помощью данного метода удалось исследовать такие специфические аспекты как: 1) насколько поздно студенты приступают к выподнению заданий иди к подготовке к экзаменам; 2) какие причины, по мнению студентов, вызывают у них состояние прокрастинации; 3) эмоциональные состояния, сопровождающие прокрастинацию; 4) как часто пропускают студенты занятия из-за невыполненных заданий; 5) что, по мнению студентов, стимулирует их на выподнение заданий. Ограниченность данного исследования состоит в том, что оно опирается на субъективный опыт студент, для повышения точности и объективности требуется исподьзование методов наблюдения и экспериментам. Диагностические методики, которые дополнили анкету, дади противоречивые результаты. Данные, полученные с помощью анкеты, показади четко разделение студентов: на тех, кто иногда находится в состоянии прокрастинации («ситуативные прокрастинаторы») и на тех, кто находится в этом состоянии перманентно («хронические»). Однако, предположение, что эти группы студентов раздичаются по уровню осмысленности жизни, не нашло подтверждения. С другой стороны, корреляционный анализ между результатами опросника «Шкала прокрастинации C. H. Lay и уровнем осмысленности жизни показал обратную значимую связь $(-0,338$, при $\mathrm{r}=0.05)$. В будущих исследования кажется целесообразным сосредоточится на экспериментальной проверке полученных с помощью анкеты результатов. Вопрос, который может быть поставлен в дальнейших исследованиях, закдючается в следующем: снизится ди степень откладывания выполнения академических заданий при условии воздействия на причины прокрастинации, которые отмечают студенты, и на сосредоточении на факторах, стимулирующих выполнение заданий.

Автор заявляет об отсутствии конфликтов интересов. 


\section{Питература}

Деонтьев, Д. А. (2000). Тест смысложизненных ориентации (СЖО). 2-е изд. М.: Смысл.

Деонтьев, Д. А. (2003). Психология смысла: природа, строение и динамика смысловой реальности. 2-е, испр. изд. М.: Смысл.

Попова, Д. И. (2020). Вдияние смысловой установки как причина ака-демической прокрастинации студентов высших учебных заведений. Северо-Кавказский психологический вестник, 18(1), С. 5-19.

Попова, Д. И., Беликова, М. Е. (2021). Академическая прокрастинация: аспекты откладывания выполнения учебных заданий студентами. Северо-Кавказский психологический вестник, 19(1), 61-77. doi: https://doi.org/10.21702/ncpb.2021.5

Blunt, A. K., Pychyl, T. A. (2000). Task aversiveness and procrastination: a multi-dimensional approach to task aversiveness across stages of personal projects. Personality and Individual Differences, 28(1), 153-167. doi: https://doi.org/10.1016/ S0191-8869(99)00091-4

Bytamar, J. M., Saed, O., Khakpoor, S. (2020). Emotion Regulation Difficulties and Academic Procrastination. Frontiers in Psychology. doi: https://doi.org/10.3389/ fpsyg. 2020.524588

Eisenbeck, N., Carreno, D. F., Uclés-Juárez, R. (2019). From psychological distress to academic procrastination: Exploring the role of psychological inflexibility. Journal of Contextual Behavioral Science, 13, 103-108. doi: https://doi.org/10.1016/j.jcbs.2019.07.007

Ferrari, J., Mason, C., Hammer, C. (2006). Procrastination as a predictor of task perceptions: Examining delayed and non-delayed tasks across varied deadlines. Individual Differences Research, 4(1), 28-36.

Ferrari, J. R., Scher, S. J. (2000). Toward an understanding of academic and nonacademic tasks procrastinated by students: the use of daily logs. Psychology in the Schools, 37(4), 359366. doi: https://doi.org/10.1002/1520-6807(200007)37:4<367::AID-PITS7>3.0.CO;2-Y

Galić, G., Matešić, K., Pačić-Turk, L. (2019). Povezanost Akademske Prokrastinacije, Akademskog Lokusa Kontrole I Osobina Ličnosti S Akademskim Uspjehom. Suvremena psihologija, 22(1), 39-52. doi: https://doi.org/10.21465/2019-SP-221-03

Gil Flores, J., De Besa Gutiérrez, M. R., Garzón Umerenkova, A. (2019). Por qué procrastina el alumnado universitario? Análisis de motivos y caracterización del alumnado con diferentes tipos de motivaciones. Revista de Investigación Educativa, 38(1), 183-200. doi: https://doi.org/10.6018/rie.344781

Gurumoorthy, R., Kumar, N. S. (2020). Study of impactful motivational factors to overcome procrastination among engineering students. Procedia Computer Science, 172, 709-717 (2020). doi: https://doi.org/10.1016/j.procs.2020.05.101

Hen, M., Goroshit, M. (2020). The effects of decisional and academic procrastination on students' feelings toward academic procrastination. Current Psychology, 39(2), 556-563 (2020). doi: https://doi.org/10.1007/s12144-017-9777-3 
Lay, C. (1986). At last, my research article on procrastination. Journal of Research in Personality, 20, 474-495.

Lee, S. Y., Hall, N. C. (2020). Understanding Procrastination in First-Year Undergraduates: An Application of Attribution Theory. Social Sciences, 9(8), 136. doi: https://doi.org/10.3390/socsci9080136

Lenggono, B., Tentama, F. (2020). Construct Measurement Of Academic Procrastination Of Eleventh Grade High School Students In Sukoharjo. International Journal Of Scientific E Technology Research, 9(1), 454-459.

Milgram, N. A., Sroloff, B., Rosenbaum, M. (1988). The procrastination of everydaylife. Journal of Research in Personality, 22(2), 197-212. doi: https://doi. org/10.1016/0092-6566(88)90015-3

Morris, P. E., Fritz, C. O. (2015). Conscientiousness and procrastination predict academic coursework marks rather than examination performance. Learning and Individual Differences, 39, 193.

Pychyl, T. A., Lee, J. M., Thibodeau, R., Blunt, A. (2000). Five days of emotion: an experience sampling study of undergraduate student procrastination. Journal of Social Behavior and Personality, 15(5), 239-254.

Rahimi, S., Hall, N. C., Pychyl, T. A. (2016). Attributions of Responsibility and Blame for Procrastination Behavior. Frontiers in Psychology. doi: https://doi. org/10.3389/fpsyg.2016.01179

Sirois, F. M. (2014). Absorbed in the moment? An investigation of procrastination, absorption and cognitive failures. Personality and Individual Differences, 71, 30-34. doi: https://doi.org/10.1016/j.paid.2014.07.016

Steel, P. (2007). The nature of procrastination: a meta-analytic and theoretical review of quintessential self-regulatory failure. Psychological Bulletin, 133(1), 65-94. doi: https://doi.org/10.1037/0033-2909.133.1.65

Steel, P., Ferrari, J. R. (2013). Sex, education and procrastination: An epidemiological study of procrastinators' characteristics from a global sample. European Journal of Personality, 27(1), 51-58.

Tice, D. M., Baumeister, R. F. (1997). Longitudinal study of procrastination, performance, stress, and health: the costs and benefits of dawdling. Psychological Science, 8(6), 454-458. doi: https://doi.org/10.1111/j.1467-9280.1997.tb00460.x

Zack, S., Hen, M. (2018). Academic interventions for academic procrastination: A review of the literature. Journal of Prevention \& Intervention Community, 46(2), 117-130.

\section{References}

Blunt, A. K., Pychyl, T. A. (2000). Task aversiveness and procrastination: a multi-dimensional approach to task aversiveness across stages of personal projects. Personality and Individual Differences, 28(1), 153-167. doi: https://doi.org/10.1016/ $\underline{\text { S0191-8869(99)00091-4 }}$ 
Bytamar, J. M., Saed, O., Khakpoor, S. (2020). Emotion Regulation Difficulties and Academic Procrastination. Frontiers in Psychology. doi: https://doi.org/10.3389/ fpsyg. 2020.524588

Eisenbeck, N., Carreno, D. F., Uclés-Juárez, R. (2019). From psychological distress to academic procrastination: Exploring the role of psychological inflexibility. Journal of Contextual Behavioral Science, 13, 103-108. doi: https://doi.org/10.1016/j.jcbs.2019.07.007

Ferrari, J., Mason, C., Hammer, C. (2006). Procrastination as a predictor of task perceptions: Examining delayed and non-delayed tasks across varied deadlines. Individual Differences Research, 4(1), 28-36.

Ferrari, J. R., Scher, S. J. (2000). Toward an understanding of academic and nonacademic tasks procrastinated by students: the use of daily logs. Psychology in the Schools, 37(4), 359366. doi: https://doi.org/10.1002/1520-6807(200007)37:4<367::AID-PITS7>3.0.CO;2-Y

Galić, G., Matešić, K., Pačić-Turk, L. (2019). Relationship of Academic Procrastination, Academic Locus of Control, and Personality Traits with Academic Success. Contemporary psychology, 22(1), 39-52. doi: https://doi.org/10.21465/2019-SP-221-03

Gil Flores, J., De Besa Gutiérrez, M. R., Garzón Umerenkova, A. (2019). Why do college students procrastinate? Analysis of motives and characterization of students with different types of motivations. Journal of Educational Research, 38(1), 183-200. doi: https://doi.org/10.6018/rie.344781

Gurumoorthy, R., Kumar, N. S. (2020). Study of impactful motivational factors to overcome procrastination among engineering students. Procedia Computer Science, 172, 709-717 (2020). doi: https://doi.org/10.1016/j.procs.2020.05.101

Hen, M., Goroshit, M. (2020). The effects of decisional and academic procrastination on students' feelings toward academic procrastination. Current Psychology, 39(2), 556-563 (2020). doi: https://doi.org/10.1007/s12144-017-9777-3

Lay, C. (1986). At last, my research article on procrastination. Journal of Research in Personality, 20, 474-495.

Lee, S. Y., Hall, N. C. (2020). Understanding Procrastination in First-Year Undergraduates: An Application of Attribution Theory. Social Sciences, 9(8), 136. doi: $\underline{\text { https://doi.org/10.3390/socsci9080136 }}$

Lenggono, B., Tentama, F. (2020). Construct Measurement Of Academic Procrastination Of Eleventh Grade High School Students In Sukoharjo. International Journal Of Scientific \& Technology Research, 9(1), 454-459. Russ.).

Leont'ev, D. A. (2000). Purpose-in-Life Test (PIL). 2nd ed. Moscow: Smysl. (in

Leont'ev, D. A. (2003). The psychology of meaning: its nature, structure and dynamics of meaningful reality. 2nd ed. Moscow: Smysl. (in Russ.).

Milgram, N. A., Sroloff, B., Rosenbaum, M. (1988). The procrastination of everydaylife. Journal of Research in Personality, 22(2), 197-212. doi: https://doi. org/10.1016/0092-6566(88)90015-3 
Morris, P. E., Fritz, C. O. (2015). Conscientiousness and procrastination predict academic coursework marks rather than examination performance. Learning and Individual Differences, 39, 193.

Popova, D. I. (2020). Influence of a meaning attitude as a cause of academic procrastination of higher education students. North-Caucasian Psychological Bulletin, 18(1), 5-19. (in Russ.).

Popova, D. I., Belikova, M. E. (2021). Academic procrastination: Aspects of postponingstudent completion of learning assignments. North-Caucasian Psychological Bulletin, 19(1), 61-77. doi: https://doi.org/10.21702/ncpb.2021.5 (in Russ.).

Pychyl, T. A., Lee, J. M., Thibodeau, R., Blunt, A. (2000). Five days of emotion: an experience sampling study of undergraduate student procrastination. Journal of Social Behavior and Personality, 15(5), 239-254.

Rahimi, S., Hall, N. C., Pychyl, T. A. (2016). Attributions of Responsibility and Blame for Procrastination Behavior. Frontiers in Psychology. doi: https://doi.org/10.3389/ fpsyg.2016.01179

Sirois, F. M. (2014). Absorbed in the moment? An investigation of procrastination, absorption and cognitive failures. Personality and Individual Differences, 71, 30-34. doi: https://doi.org/10.1016/j.paid.2014.07.016

Steel, P. (2007). The nature of procrastination: a meta-analytic and theoretical review of quintessential self-regulatory failure. Psychological Bulletin, 133(1), 65-94. doi: https://doi.org/10.1037/0033-2909.133.1.65

Steel, P., Ferrari, J. R. (2013). Sex, education and procrastination: An epidemiological study of procrastinators' characteristics from a global sample. European Journal of Personality, 27(1), 51-58.

Tice, D. M., Baumeister, R. F. (1997). Longitudinal study of procrastination, performance, stress, and health: the costs and benefits of dawdling. Psychological Science, 8(6), 454-458. doi: https://doi.org/10.1111/j.1467-9280.1997.tb00460.x

Zack, S., Hen, M. (2018). Academic interventions for academic procrastination: A review of the literature. Journal of Prevention E Intervention Community, 46(2), 117-130. 